\title{
Filigrane
}

Écoutes psychanalytiques

\section{Bisexualité et différence des sexes}

\section{Catherine Chabert}

Volume 25, numéro 1, printemps 2016

Actes de la journée clinique de la SPQ (Catherine Chabert)

URI : https://id.erudit.org/iderudit/1037377ar

DOI : https://doi.org/10.7202/1037377ar

Aller au sommaire du numéro

Éditeur(s)

Revue Santé mentale au Québec

ISSN

1192-1412 (imprimé)

1911-4656 (numérique)

Découvrir la revue

Citer cet article

Chabert, C. (2016). Bisexualité et différence des sexes. Filigrane, 25(1), 103-118.

https://doi.org/10.7202/1037377ar d'utilisation que vous pouvez consulter en ligne.

https://apropos.erudit.org/fr/usagers/politique-dutilisation/ 


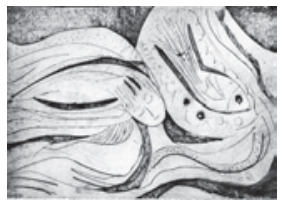

\section{Bisexualité et différence des sexes ${ }^{1}$}

\section{Catherine Chabert}

e souhaite tout d'abord vous remercier chaleureusement de m'avoir invitée et en particulier Louise Mercier qui a organisé cette rencontre avec amitié et efficacité. C'est toujours un honneur et un plaisir pour moi de venir à Québec, et je garde un souvenir précieux des précédentes rencontres qu'il m'a été donné de faire ici. C'est aussi, comme chaque fois, l'opportunité de parler de clinique et de théorie avec la liberté plus grande offerte par l'extratéritorialité, même si, en dépit des distances géographiques, nous sommes très proches par nos affinités de pensée et de sensibilité.

Avant de m'engager dans mon exposé, je voudrais rapidement tracer mon trajet depuis le début de la préparation de ma conférence d'aujourd'hui. Un premier mouvement m'a portée jusqu'à l'écriture de l'argument que je vous ai envoyé. Mais dans la suite, j'ai été amenée à modifier en partie ma trajectoire, si bien que la seconde partie s'éloigne un peu de l'itinéraire initial... sans perdre de vue pour autant l'essentiel, en tout cas je l'espère.

Je vais donc comme prévu parler de la bisexualité psychique et de ses destins dans la cure et me centrer ensuite essentiellement sur sa part féminine et sur certains de ses écueils dont on peut penser qu'ils sont, eux aussi, produits par la bisexualité: la question du féminin pur, à partir des travaux de Winnicott d'abord, puis celle du surmoi au féminin chez les deux sexes.

En commençant sa conférence sur la féminité (1932), Freud insiste d'emblée sur l'existence et le maintien de la bisexualité psychique: bisexualité qui témoigne de l'inscription des deux sexes, masculin et féminin, chez tout individu; psychique, pour rendre compte du caractère interne de cette inscription. On peut donc s'interroger d'abord sur la place et la fonction de la bisexualité dans la construction de la réalité psychique et considérer, en allant un peu vite, que la réalité psychique est un lieu et un objet de déformation en contraste avec la réalité objective qui, elle, se situe au plus près de la perception: elle désigne un ensemble relativement identifié dans les oppositions classiques entre réalité interne/réalité externe, dedans/dehors, 
monde interne/monde externe et encore, pour reprendre le propos d'André Beetschen, sur lequel j'aurai l'occasion de revenir, l'intime et l'étranger.

Revenons très brièvement aux articulations de la psyché et de la différence des sexes chez Freud: sa thèse est celle d'une différence anatomique ou biologique dont les conséquences psychiques se développent à partir de la perception de ces différences, puis, essentiellement avec le complexe d'Eedipe et les remaniements après-coup de ces perceptions. Le biologique constitue un ancrage "de fait», un roc dira-t-il plus tard, sur lequel s'étayent les différences psychiques: l'écart entre les deux sexes se formule en termes de "avoir ou ne pas avoir », et cette dissymétrie se déplace, se transfère en dissymétrie psychique déterminante quant aux destins de la psychosexualité. Il s'agit donc d'approfondir et d'analyser le devenir d'un principe essentiel de la psychanalyse, qui place la différence des sexes au fondement de la psyché et dans sa dynamique, dans son intimité la plus absolue. Il s'agit de se défaire des tendances qui situent trop concrètement la sexualité dans la réalité des engagements relationnels de son exercice et oublient ses composantes psychiques inconscientes, dès les commencements, en négligeant la vivacité voire la violence des conflits internes qu'elle fomente. On peut considérer «la chose sexuelle» comme une composante essentielle de la réalité psychique, ou encore comme un de ses produits: dès les débuts de la vie, la double valence du masculin et du féminin s'engage dans cette construction.

Comme Beetschen le souligne, la bisexualité n'est pas un hâvre (2016), elle peut tout au plus offrir un relais lorsque la différence des sexes est éprouvée dans l'excès d'excitation ou de douleur: chaque fois qu'une différence contraint, le surinvestissement de son contraire s'impose du fait de l'attaque narcissique que celle-ci implique, mais en elle-même cette opération reste violente par les mouvements de bascule qu'elle impose.

La bisexualité se situe en effet à la fois aux origines narcissiques de la psychosexualité et comme héritière du complexe d'Edipe: elle peut être emportée par un moi idéal en quête d'unité et de fusion ou engagée dans un procès de différenciation susceptible d'appréhender la part de l'un et de l'autre, et l'hétérogénéité d'empreintes séparatrices du même. Ce sont ces deux polarités qui tantôt alternent, tantôt se condensent dans les précipités d'identifications issues de la nécessité du choix d'objet et de ses avatars.

Il est sûr qu'au-delà de ses racines, la bisexualité trouve ses effets dans les éléments communs qui soudent les identifications sexuelles. «La bisexualité de tous les humains» déclarée par Freud dès 1896, se retrouve régulièrement et notamment en 1932, dans la Conférence sur la féminité, qui signale 
l'impossibilité de s'approcher du masculin ou du féminin sans l'inscrire dans son rapport à l'autre sexe: les deux sont liés, intrinsèquement, et liés, paradoxalement, par la différence. Or la logique de la différence soutient et affirme, au-delà de l'altérité, l'existence et la reconnaissance d'objets internes pris dans les réseaux de la sexualité, quels qu'en soient les registres: partiellement défaite de ses rouages narcissiques, celle-ci s'éloigne d'une identification en miroir; la logique de la différence relève davantage de la projection de l'amour et de la haine, elle prend l'objet en compte dans une démesure impressionnante pour s'en emparer ou pour s'en déprendre, radicalement. L'angoisse et l'inquiétant, tels que les construit André Beestchen, peuvent s'attacher au sexe féminin, à l'effroi lié à l'angoisse de castration et de pénétration, comme il le propose; mais on pourrait aussi penser l'inquiétant dans le mélange que la bisexualité induit, dans l'emmêlement des deux sexes, le familier et l'étranger, le connu et l'inconnu, généré par le fantasme et la sexualité infantile.

Le complexe d'CEdipe, quant à lui, ne peut s'entendre seulement dans ses configurations les plus structurantes: la référence à la différence des sexes et des générations ne signifie pas que celle-ci soit psychiquement admise sans hésitation. L'infantile est sexuel et le demeure, il ne se cantonne jamais uniquement dans les zones les plus troublées de l'indifférenciation, il affronte inévitablement la différence et s'en accommode plus ou moins. La bisexualité, cette immense construction freudienne, est toujours aussi vivace et ne bascule que par «un plus» de l'un ou de l'autre, elle ne signifie pas la confusion des sexes, elle signale l'existence des deux, masculin et féminin, et leurs configurations singulières et plurielles.

Revenons au masculin et au féminin, et d'abord, une fois de plus, au père et à la mère: la prévalence accordée à l'une ou l'autre figure caractérise des courants de pensée considérés parfois comme conflictuels voire irréductibles. Être, là encore, du côté de l'un ou de l'autre? La langue, la règle, la séparation, l'interdit, la sexualité, le père? Ou la parole, l'excitation, la contenance, la confusion, la perte, la toute-puissance, la mère? Le cortège des représentations et des concepts qu'elles suscitent pour mettre en mots l'expérience, suit immanquablement les mêmes tracés pour retrouver la racine paternelle ou maternelle. Comme si, hors de la grande dramaturgie odipienne, il n'y avait pas de lieu possible pour rassembler le père et la mère, comme si la pensée de leur coexistence ou plutôt de leur cohabitation se révélait intolérable, du fait de la douleur qu'inflige la reconnaissance de leur vie commune, de leurs liens, de leurs désirs. Une évidence d'une grande 
simplicité: être du côté de l'un ou de l'autre revient à les séparer, et donc à éviter de les voir ou de les penser ensemble lorsque l'attente se confond avec la solitude et que celle-ci bascule dans la détresse. C'est bien cet état de détresse que les positions névrotiques tentent de bannir: ne pas accepter la différence pourrait constituer un jalon indispensable pour éviter d'être confronté aux désirs et à l'excitation qu'ils suscitent. C'est la mise à l'écart de la scène primitive, quels qu'en soient les partenaires, qui génère la douleur, et l'impossibilité de la supporter qui contraint à surinvestir la bisexualité, ce qui assure alors une protection contre l'horreur de l'exclusion : être hors du désir de l'autre, être exilé de la terre maternelle ou paternelle, être banni de leurs corps et de leur commerce amoureux.

L'autre obstacle apparaît dans le conflit lié au choix d'objet: faut-il choisir? Entre le père et la mère, faut-il toujours privilégier l'un plutôt que l'autre, faut-il abandonner l'un au bénéfice de l'autre? Faut-il encore se laisser prendre par la question vive posée à l'enfant dans sa maladresse brutale: "Qui préfères-tu, ton père ou ta mère? Qui aimes-tu le plus, elle ou lui?», question à laquelle, selon Freud, l'obsessionnel ne peut jamais répondre (Freud, 1909). On peut penser alors que le doute obsessionnel se noue justement là, à l'intersection du choix d'objet, des identifications et du maniement pulsionnel rendant particulièrement compliquées les liaisons et les déliaisons de l'amour et de la haine. Ne sommes-nous pas confrontés alors à la force exemplaire d'une réalité psychique puissante dont les effets délétères peuvent entraver l'ensemble du fonctionnement mental?

Les deux patients dont je vais parler, un jeune homme et une jeune femme, sont clairement pris par cette impossibilité de choisir, de se décider dans leur vie amoureuse, et s'ils ne s'accommodent pas de cet état, c'est qu'il engendre et irradie une souffrance extrême menaçant d'autres domaines de leur vie. Ils ont le même âge, et ont par hasard commencé leur analyse en même temps. Ils présentent l'un et l'autre les caractéristiques fiables de la névrose obsessionnelle, et de leur différence, sexuelle.

Tous les deux ont dépassé l'âge de l'adolescence et sont entrés dans leur vie d'adulte: et pourtant se pérennise, chez l'un comme chez l'autre, une problématique très caractéristique de la réactualisation œedipienne reconnue à cette période de la vie. Ils sont tous les deux de brillants intellectuels, aussi modestes l'un que l'autre; ils n'ont jusqu'ici aucune difficulté dans leur jeune vie professionnelle qui leur convient parfaitement. Leur souffrance est ailleurs: pour lui, la découverte, soudaine, récente, de pouvoir être profondément blessé et meurtri par la rupture d'une liaison amoureuse 
particulièrement houleuse; pour elle, l'impossibilité, encore récemment répétée de dire oui à un homme qu'elle aime, et de réaliser ses désirs d'enfant.

Les oscillations ordonnées par un doute obsessionnel patent trouvent des formes différentes chez l'un et chez l'autre: lui, semble découvrir en même temps que moi, qu'il a toujours été pris entre deux filles, puis deux femmes; elle, a rompu une promesse de mariage quelques jours avant la cérémonie tant elle était envahie par des doutes concernant son amour pour son promis. Doutes identiques qui l'assaillent aujourd'hui et l'empêchent de s'engager avec son partenaire actuel.

Les modalités d'instauration du transfert sont, elles aussi, différentes: lui, très réservé, garde ses distances, s'oblige à partir au loin, se cantonne dans les remous récents et bouleversants de sa dernière liaison pour essayer d'en comprendre les ressorts, essentiellement d'ailleurs ceux qui pourraient rendre plus compréhensible le fonctionnement de son amie, mais j'entends là une interrogation taraudante quant à la part du féminin chez lui.

Elle, s'emporte d'emblée dans un transfert massif, elle est constamment occupée par ses séances, elle rêve beaucoup, arrive toujours très émue à ses séances, elle est, à son insu, envahie par la crainte de trahir sa mère en me parlant trop.

Ils ont en commun d'avoir passé leur adolescence loin du foyer familial, et chacun en reconstruit l'histoire: lui, se dit heureux d'avoir échappé ainsi aux disputes conjugales constantes de ses parents, et c'est au cours de ces années qu'il a pu découvrir à la fois la force des liens d'amitié et sa vocation créatrice. Elle, nie les effets de ces longues séparations car elle avait été trop marquée par les nombreux déménagements de sa famille du fait des activités professionnelles de son père. Elle se réjouissait d'habiter au même endroit, d'avoir un port d'attache, ce qui lui permettait, à son tour, de voyager de par le monde pour son travail.

Lui, revendiquait, sous des dehors relativement impassibles, une forte sensibilité qui aurait échappé à ses parents, surtout à sa mère. Elle, déplorait sa facilité à s'émouvoir et à pleurer, alors qu'elle se sentait à la fois puissante intellectuellement et fondamentalement autonome: le conflit pour elle se nouait précisément là, elle voulait "être mère» mais à travers ses motifs manifestes de résistances à ce désir, j’entendais bien sa revendication masculine, son désir d'être le maillon fort dans sa fratrie, et l'usage de cette identification masculine qui lui permettait sans réserve de séduire son père car elle voulait «être à sa hauteur »! Ce même père qui demandait aux garçons qu'elle lui présentait: «Serez-vous à la hauteur de ma fille?» 
Chez l'un comme chez l'autre, le recours à la bisexualité psychique avait constitué un appui considérable, mais dans l'avancement de la vie d'adulte, son maintien constituait un obstacle majeur, sans doute parce qu'il était sous-tendu par un attachement démesuré et puissant aux figures parentales: pour elle, c'était une évidence, elle offrait de sa famille et de ses parents des représentations extrêmement idéalisées. Chez lui, au contraire, la conviction était forte d'être très éloigné de ses parents, psychiquement, affectivement, culturellement.

Après quelque temps d'analyse, je pus observer un changement inversé qui les rapprochait curieusement pour moi: elle commençait à entrevoir les failles (et la faillite) de son idéalisation initiale et découvrait avec rage les brèches, les défauts, les écarts, les erreurs de sa mère en particulier. Lui, fut pris au dépourvu, au décours d'un rêve, par la place de sa mère dans son enfance, il retrouva des souvenirs de tendresse extrême, des photos d'elle, jeune et jolie, le tenant dans ses bras, il avoua qu'en vérité, et contrairement à ce qu'il en avait dit jusqu'ici, elle s'intéressait beaucoup à ce qu'il faisait et était très fière de son aîné.

Deux autres points encore: il se souvint de sa jalousie à l'égard de son frère, celui qu'il appelle «le fils de mon père», des coups qu'il lui donnait, des blessures qu'il lui infligea, de sa honte et de sa culpabilité vis-à-vis de ce cadet pour lequel il nourrit des sentiments de dérision tendre cachant probablement une haine «indestructible». Elle, rêva que son frère périt dans un naufrage en mer et lutta contre l'idée qu'il pourrait être un rival envié. Elle était partout la première.

Et enfin, un dernier commentaire à propos de leurs relations amoureuses: lors de ma première rencontre avec lui, j'avais remarqué sur son visage, une légère blessure. Plus tard, je compris qu'en effet sa compagne, après une exhibition publique blessante qu'il avait tenté de freiner, l'avait giflé et que c'était l'angoisse associée à cette scène, l'image de lui-même qu'elle lui imposait, faible, châtré (comme une femme qui saigne?), qui l'avait déterminé à s'engager dans l'analyse. Il y découvrit la force de son masochisme, et bien sûr, de manière concomitante, du sadisme sous-jacent à son extrême gentillesse, certes, mais aussi fortement présent dans sa manière de vivre entre deux femmes sans les choisir mais en les faisant souffrir de ce non-choix.

Quant à elle, si calme et raisonnable et généreuse, si contrôlée aussi dans ses mots et ses gestes, si facilement rattrapée par le remords, le scrupule, l'autoreproche, elle finit par faire part des scènes violentes qu'elle imposait 
à son ami, au cours desquelles son sadisme se déchaînait dans des propos mordants, hostiles, blessants, dont elle maintenait l'argumentaire pendant les séances si bien que je pouvais sans difficulté penser qu'elles m'étaient aussi adressées.

Pour l'un et pour l'autre, au cours de cette première année de l'analyse, le transfert suivit des voies parallèles et dissymétriques: elle, se dessaisit de son idéalisation transférentielle initiale et lui, aborda timidement les mouvements tendres d'un transfert amoureux. Cependant, ces modifications nécessitèrent très vite un retour en force de ce qui contrait l'indéfectible attachement - odipien sans doute, mais pas seulement - aux figures parentales (dans sa double valence aimante et haineuse), un retour massif de leur bisexualité psychique renforçant leurs identifications: elle s'engagea dans un discours féministe militant; mais surtout mit en évidence ce qu'elle cachait depuis fort longtemps sous son extrême modestie et ses doutes permanents: la conviction d'être la plus forte, grâce à son intelligence et à son identification à son père, son seul interlocuteur valable. Et en même temps, sa féminité se développait dans sa présentation physique, et aussi dans la reconnaissance d'une appartenance au monde des femmes dont elle se disait à la fois fière et soulagée. Mais sa furie contre son ami reprit de plus belle, sans doute à la mesure de son attachement pour lui, elle mit leur relation en danger et sembla se décider pour un destin de femme solitaire, forte et indépendante, échappant définitivement au pouvoir des hommes.

Quant à lui, après avoir longtemps élaboré sa rupture avec sa violente amie, il utilisa des vacances d'analyse pour renouer avec elle, dupé par luimême dans l'espoir d'un changement, et assurant, avec force arguments, que cette fois il ne se laisserait pas faire! Un régime plus tempéré lui permit de poursuivre quelques mois cette relation et, là encore, la bisexualité psychique vint à son secours: d'un côté, il assurait sa virilité avec assurance et efficacité, mais de l'autre, insidieusement, il était aux prises avec un masochisme féminin évident pour moi.

Ce faisant, l'un et l'autre croyaient - illusoirement - ne rien perdre, et, dans le récit permanent de leur vie amoureuse, ils me plaçaient en témoin impuissant de scènes primitives où ils occupaient une place mobile mais constante: c'était moi, l'enfant à la fois exclu et spectateur indispensable, l'enfant excité et triste, l'enfant abandonné par un couple entièrement capté par le désir et la quête de sa satisfaction. En de telles occurrences, c'est la réalité psychique dans le matériel des séances et dans l'adresse transférentielle qui soutient le processus: cette assignation transférentielle - me mettre 
dans la position de l'enfant qu'ils avaient été, qu'ils étaient toujours, dans une déformation majeure des identifications de la situation analytique constituait la voie de changements potentiels.

Car l'envers de la bisexualité, ce contre quoi elle s'érige dans les cures d'adultes en occupant toutes les places, put advenir au décours d'interprétations: l'impuissance et la détresse surgirent avec une force qu'aucun des deux ne put anticiper. Lui, sombra dans le désespoir de son adolescence solitaire, ses constructions psychiques concernant son détachement, son indépendance voire son indifférence vis-à-vis de ses parents s'effritèrent, le laissant désemparé et sans ressources. Elle, retrouva la petite fille insomniaque, débordée par ses angoisses de mort, elle se souvint de ses larmes de nuit, et le vaillant petit soldat qu'elle s'était forgé s'évanouit avec elles.

J'en viens maintenant à une autre question plus précise: de quel féminin parle-t-on lorsqu'on examine le féminin chez les deux sexes? Peut-on l'entendre essentiellement dans les identifications primaires qui constitueraient le socle de tout le processus de construction identitaire, ou bien davantage du côté des identifications oedipiennes?

Une question subversive surgit concernant la position de Winnicott à propos de la bisexualité (1966): c'est le clivage entre masculin et féminin qu'il défend, pour donner corps au féminin pur, exempt de toute référence à la différence, fondant en un seul l'enfant et le sein, et, à partir de cette unité, l'essence même de l'être (being). Mais ce féminin pur n'a rien à voir avec la pulsion, il est «non-contaminé», "distillé» et conduit à l'être, seule base de la découverte du self et du sentiment d'exister. L'exposé clinique qui accompagne le propos de Winnicott est extrêmement connu: il rapporte la cure d'un homme, déclarant qu'il était pris par son «envie du pénis», à quoi Winnicott avait réagi par cette phrase célèbre: «Je suis en train d'écouter une fille. Je sais parfaitement que vous êtes un homme, mais c'est une fille que j'écoute, et c'est à une fille que je parle.» Cette séquence devrait illustrer parfaitement la position de Winnicott quant au clivage des éléments masculins et féminins chez l'homme et chez la femme. Elle montre aussi sa conception et sa pratique du transfert: le patient imagine qu'on le prendrait pour un fou s'il parlait de cette fille... et Winnicott poursuit: «C’est moi qui vois la fille et qui entends une fille parler alors que c'est un homme qui est sur mon divan.»

Une première remarque concerne le fameux clivage entre masculin et féminin, et la lutte interne violente entre la voix de la fille qui veut à tout prix être entendue et reconnue et les défenses de l'homme qui érige un barrage puissant pour ne pas la laisser passer. Cette idée du clivage nous éloigne de la 
bisexualité psychique telle que Freud l'a construite, dans laquelle, il n'y a pas de coupure drastique entre les deux, mais seulement «bien plus de l'un que de l'autre», comme il l'écrit dans la conférence sur la féminité, une position beaucoup plus dialectique, qui, scandaleuse à son époque, est devenue, audelà du champ de la psychanalyse, presque un principe, sinon une revendication. Cependant, la relecture du commentaire de Winnicott me fait douter de l'existence d'un clivage effectif: le combat entre les représentations opposées, la bataille qui dresse l'un contre l'autre ne doivent-ils pas être entendus comme un conflit? Ne retrouverait-on pas dans cette bataille, au-delà du masculin et du féminin, les traces inextinguibles du rapport entre homme et femme, entre père et mère? Et finalement, l'actualisation d'une scène primitive condensant toujours identification et choix d'objet?

Je reviens à Pontalis (1973) : il souligne les deux points de butée auxquels se heurte Freud lorsque, à la fin de sa vie, il cherche les raisons des échecs thérapeutiques de la psychanalyse: «l'un, théorique, qui lui fait invoquer en dernier recours l'idée d'une "inclination" (Neigung) au conflit» comme facteur spécifique (manifestation de la pulsion de mort?); l'autre, auquel achoppe l'expérience: pourquoi l'homme a-t-il tellement peur d'être une femme $[\ldots]$ ? Pourquoi la femme renonce-t-elle si difficilement — voire ne renonce jamais — à son désir d'avoir, d'obtenir, le pénis? C'est le roc (p. 19). Il existe une correspondance évidente, nous dit Freud, entre les deux thèmes, le refus chez l'homme, la revendication chez la femme: "Quelque chose, qui est commun aux deux sexes, a été modelé, du fait de la différence entre les sexes, en des formes différentes d'expression.» (Freud, 1937, p. 53).

Il faut souligner cette double part de la théorie et de l'expérience irréductibles à l'analyse, et surtout l'inclination au conflit qui est généralement passée sous silence chaque fois que «le roc» est évoqué: celui-ci est généralement réduit au refus de la féminité pour les deux sexes, et oublie la différence clairement exposée par Pontalis, ne pas vouloir être une femme ou ne pas renoncer à avoir un pénis. La tendance actuelle à surinvestir le narcissisme trouve ses effets dans l'opération qui ramène au même les deux positions de l'homme et de la femme alors que le point de vue freudien est infiniment plus subtil: la bisexualité commune trouve des voies d'expressions différentes. Les positions perverses jouent de cette ambiguïté dans le traitement de la bisexualité: au regard de la perversion, la femme a un pénis et l'homme veut être une femme! L'inclination au conflit disparaît car, quelle que soit la prévalence d'une identification ou d'une autre, les deux, le masculin et le féminin sont toujours présents au sein du même. 
Ma seconde remarque à propos de Winnicott prend la suite de mes interrogations: il a trait au féminin pur, saisi dans l'enfant au sein, «le bébé devient le sein (ou la mère), l'objet est alors dans le sujet» (Winnicott, 1966, p. 308) : cet «objet subjectif» est le premier objet, «l'objet qui n’a pas encore été répudié en tant que phénomène non moi» (p. 309). Le sentiment qui l'accompagne, dit Winnicott, est antérieur à être-un-avec, parce qu'il n'y a rien eu d'autre que l'identité: le moi commence à s'organiser et le mode de relation objectale de l'élément purement féminin établit «la plus simple des expériences", l'expérience d'être.

Position acceptable ou discutable? Peut-on admettre une mère défaite de la part sexuelle de son identité? Oui, dit Winnicott du fait du clivage inaugural, radical entre l'élément masculin et l'élément féminin. Peut-on admettre, hors du fantasme qui s'en nourrit, une captation complète de la mère par l'enfant, sans aucune référence, même à l'ombre d'un père? Et quand j'évoque «le père», je pense au père de l'enfant, bien sûr, mais aussi au père de la mère. Sans aucune trace de cette présence, sans aucun effet de cette existence? Comment associer, alors, ce point de vue et la conception du soi, compris dans l'entre-deux, entre l'enfant et la mère, entre dedans et dehors, entre moi et non-moi, entre l'intime et l'étranger? Bien sûr, dans une perspective développementale ou psychogénétique, cette unité radicale mère-enfant est transitoire et se défait progressivement dans le temps. Mais il arrive que ces constructions soient fixées, inamovibles, et se maintiennent grâce à un clivage extrêmement efficace: le clivage entre le masculin et le féminin double le clivage du moi et assure la coexistence de la croyance inébranlable dans un lien unique avec la mère et de la reconnaissance d'une configuration triangulaire formellement construite.

Le clivage entre le masculin et le féminin, lorsqu'il touche un homme ou une femme, en adoptant une logique d'exclusion qui consiste à écarter l'un ou l'autre, a comme premier effet de maintenir un système éminemment narcissique, déniant tout signe de différence parce que celle-ci appelle trop vite l'effondrement d'une unité dont la préservation constitue une préoccupation première: quelque chose qui s'inscrit dans une pulsionnalité autoconservatrice, luttant âprement contre tout surgissement d'un sexuel menaçant parce que séparateur du même. À cet égard, le rabattement de la perversion sur le narcissisme risque de faire perdre de vue autre chose, constamment maintenu dans la perversion, à savoir le lien à un objet qui doit rester distinct, qui doit assurer sa fonction fétichique, garant inanimé de la vitalité du moi. Au-delà, lorsqu'il prend ses sources dans une bisexualité 
ayant abandonné ses supports psychiques pour s'attacher compulsivement aux perceptions qui en donneraient la preuve sensible, le clivage est le produit d'une position perverse et non pas seulement narcissique. Cela dit, on peut se demander dans quelle mesure cette position "perverse» n'est pas inéluctablement convoquée dans l'actualisation transférentielle, à l'ombre de la perversion polymorphe de la névrose infantile.

Je me demande si, parmi les écueils du féminin susceptibles d'être convoqués, celui associé à ce que Winnicott appelle «le féminin pur» n’occupe pas une place majeure: si on l'entend comme un produit de la désexualisation maternelle, il peut accueillir nombre de fantasmes et de souvenirs refoulés du fait de l'interdit de l'inceste d'abord. Mais ce féminin pur peut aussi incarner un moi idéal défendu farouchement par un surmoi sévère qui empêche l'accès au plaisir et à la jouissance sexuelle. Actif chez les hommes et chez les femmes, ce féminin pur est susceptible cependant d'apparaître dans des formes différentes: le maintien à tout prix de l'idéalisation de la mère ne produit pas les mêmes effets. Je reviens à mes deux patients, pour tenter d'approcher les destins de cette figure idéale au cours de l'analyse.

Chez ma patiente, je l'ai déjà évoqué, la désidéalisation apparut d'abord dans le transfert dès que la frustration surgit à propos d'un manquement banal aux règles de l'analyse. Ce que la jeune femme n'acceptait pas, c'est que je respecte et lui demande de respecter ces principes fondamentaux auxquels nous devons tous nous plier. Apparut chez elle l'attente d'être traitée différemment, d'être une patiente à part, et finalement une fille préférée: les privilèges accordés par sa mère à son petit frère malade, sa déception de ne pas être l'objet d'amour unique de sa mère purent trouver une forte compensation dans l'érotisation via le travail intellectuel de la relation avec le père. Petite fille douée, elle devint une lycéenne et puis une étudiante extrêmement brillante, laissant loin derrière elle tous ses rivaux et d'abord son frère. La réalisation de ses désirs incestueux à travers la sublimation n'en restait pas moins source d'une culpabilité immense vis-à-vis de la mère qui, elle, n'avait aucune activité intellectuelle ou artistique mais consacrait sa vie à la religion et aux oeuvres caritatives. Le «féminin pur» occupait une place essentielle à travers le culte de la Vierge.

Chez mon patient, la configuration était bien différente: ses souvenirs d'enfance étaient saturés de scènes violentes entre ses parents, scènes de coups dans lesquelles les places de l'un et de l'autre, de la victime et du bourreau n'étaient pas clairement définies et qui rendaient compte plutôt d'une excitation extrême et exhibitionniste. Le féminin pur s'incarnait pour lui dans 
l'une des composantes de ses choix amoureux: la femme tranquille, douce et apaisante qui pendant longtemps apparut comme une figure de contreinvestissement d'une mère pulsionnelle. Chez lui, donc, se retrouvaient avec évidence les caractéristiques décrites par Freud d'un type particulier de choix d'objet chez l'homme, en ce sens qu'en effet, il s'engageait dans des liaisons amoureuses passionnelles avec des femmes «de petite vertu» tout en maintenant des liens de grande tendresse avec celle qui, depuis de longues années, incarnait l'idéal d'un féminin pur exemplaire.

Chez l'un comme chez l'autre, c'est bien le risque de rupture du lien amoureux qui permit le renoncement — partiel — à une bisexualité psychique essentiellement soutenue par le désir de conserver l'identification à l'autre sexe. Chez elle, le refus de renoncer à une revendication phallique commença à s'émousser lorsqu'elle prit conscience de la perte que la rupture amoureuse impliquait: renoncer à être une mère, renoncer à être l'objet d'amour d'un homme pour rester fixée à une place infantile, définitivement. Car se séparer de son ami signifiait pour elle qu'elle ne pourrait jamais partager sa vie avec un homme, et qu'elle n'aurait jamais d'enfant: c'était le prix à payer pour rester la fille amoureuse de son père, la punition pour le maintien de son lien incestueux.

Chez lui, les choses étaient plus compliquées: en dépit de la répétition en série de ses choix amoureux, il découvrit son attente, immense, d'une relation à la fois tendre et sexuelle avec une femme sans pouvoir pour autant la satisfaire. Il fut envahi par des fantasmes de scène primitive au sein desquels la femme et l'enfant se confondaient dans une identification masochiste extrêmement douloureuse: pris entre la femme battue et l'enfant maltraité, il perdait son identité d'homme.

Chez l'un comme chez l'autre, la haine était massivement refoulée, isolée puis renversée en son contraire: associée, par la voie du sadisme, à une excitation à la fois envahissante et difficile à admettre, elle engendrait une culpabilité dévastatrice en se retournant massivement contre le moi.

«Le tableau clinique de la mélancolie fait ressortir l'aversion morale du malade à l'égard du moi propre, avant l'étalage d'autres choses », écrit Freud en 1915 qui en analyse le mécanisme: les autoreproches que le sujet s'adresse à lui-même sont destinés, à l'origine, à l'objet aimé décevant et, en fait, le visent et l'atteignent grâce à la régression narcissique. Si l'angoisse de mort est tellement forte dans la mélancolie, c'est que le surmoi n'y assure pas sa fonction de protecteur: être aimé du surmoi, même au minimum, est la condition pour pouvoir rester en vie, car le surmoi assure, par identification 
et intériorisation, un regard bienveillant pour le moi, à l'instar de celui du père qui interdit parce qu'il aime et protège. Mais le surmoi est aussi une formation du moi issue de l'œdipe ou, au moins, consolidée par lui: il relève, comme toute identification, d'une sédimentation à partir de laquelle un double mouvement s'accorde - identification au père et à la mère dans le cadre d'un œdipe complet si bien que «cette modification du moi garde sa position particulière, elle s'oppose au reste du contenu du moi comme idéal du moi ou surmoi» (Freud, 1915, p. 246).

Double identification dans la constitution du surmoi: l'une qui s'ancre sur l'état d'impuissance et de faiblesse du moi de l'enfant, en quête de protection; l'autre arrimée au développement de l'œedipe, réclamant, elle aussi, un interdit préservant de la confusion incestuelle et permettant un compromis effectif entre la frustration et la satisfaction du désir.

Ainsi, la capacité à renoncer sans être détruit peut être considérée comme un effet de la fonction protectrice du surmoi, de son effectivité. Ce destin de la culpabilité met en évidence la part vivante du moi, son empreinte libidinale indéfectiblement attachée à l'objet, mais il arrive que le renoncement soit particulièrement difficile à admettre, même dans des contextes où le narcissisme et la mélancolie apparaissent de manière ponctuelle. N'oublions pas en effet que la force du surmoi se mesure au poids de l'énergie pulsionnelle et des désirs qu'elle nourrit: plus les désirs sont vifs, plus le surmoi est sévère. On peut penser alors que la sauvagerie tyrannique du surmoi est en quelque sorte consubstantielle de l'impact incestueux de l'œdipe et du maintien d'une bisexualité essentiellement mobilisée par la nécessité de ne pas choisir, pour ne pas avoir à perdre ou à renoncer. C'est un surmoi despotique et cruel qui surgit alors dans la condensation des rôles - du criminel et du bourreau. Et c'est sans doute la part de plaisir soustraite par le scénario masochiste qui continue, tant qu'elle reste vivante, de nourrir la fureur du surmoi.

Même si Freud tient compte de la bisexualité psychique et des croisements qu'elle détermine, il est une instance psychique pour laquelle il souligne le «bien plus de l'un que de l'autre»: le surmoi des hommes s'oppose à celui des femmes parce que les formes de l'oedipe et partant, de son déclin ne sont absolument pas les mêmes. Chez le garçon, l'angoisse de castration, menace de punition pour la transgression incestueuse et parricidaire, contraint à renoncer aux souhaits œedipiens, et ce renoncement est la condition et la garantie d'une mesure de protection à la fois narcissique et objectale. 
Chez la fille, le complexe de castration pousse vers le père et prépare à l'œdipe (au lieu de le détruire, comme chez le garçon). La petite fille reste longtemps dans l'œedipe et elle ne l'abolit que bien plus tard, si bien que la formation de son surmoi pâtit de ces circonstances: il ne peut acquérir «la force et l'indépendance qui lui confèrent sa significativité culturelle» (1932, p. 213).

On pourrait penser, en soulignant le trait, que le déclin de l'œdipe trouve son modèle «idéal» chez le garçon alors que chez la fille, l'indétermination, l'inachèvement du complexe nucléaire offre, en contraste, une illustration patente des destins pathologiques du même complexe et des formes tout aussi contrastées du surmoi chez l'un et l'autre sexe. Mon hypothèse est que, du fait de la bisexualité psychique, le surmoi «au féminin» est susceptible de se découvrir tout autant chez les hommes, soit comme une composante partielle et ponctuelle, soit dans une inscription durable, reconnaissable comme un «trait» d'identification majeur.

Je reviens rapidement, à ce sujet, vers mes deux patients et aux développements récents de leur analyse. Lui d'abord: alors qu'il s'est engagé dans une relation amoureuse à la fois plus stable et plus "complète», qu'il peut se confronter à l'idée d'être père et aux difficultés qu'elle engendre, il est saisi par une absence de désir pour sa compagne qui le fait brusquement douter de son amour pour elle. Il décide très brutalement de rompre et m'annonce en même temps qu'il a décidé de partir un mois pour terminer un travail important «au vert». Comme d'habitude, il ne paie pas les séances manquées.

Elle, doit se marier dans quelques mois et essaie de concrétiser son désir d'enfant. Et tout à coup, ses doutes surgissent dans un emballement démesuré qui la poussent à vouloir tout arrêter (sauf l'analyse). Cet épisode surgit dans une période où elle déclare avoir perdu sa confiance en moi, à propos des séances manquées.

Ces deux patients partagent un rapport particulier à cette règle de l'analyse: tout en étant extrêmement respectueux des principes de l'analyse, ils achoppent très précisément sur le paiement des séances manquées. C'est là que se noue un conflit transférentiel violent chez elle, retenu et comme abrasé chez lui. Chez l'un comme chez l'autre, les autocritiques reviennent avec un acharnement qui montre bien la grande difficulté dans laquelle ils se trouvent de reconnaître la part agressive d'un transfert négatif insidieux et tenace, évidemment très marqué par la déception et la frustration.

C'est en référence à un surmoi cruel, un surmoi au féminin, que je comprends à la fois la transgression et la punition majeure qu' elle engendre: ne pas 
pouvoir véritablement s'approprier les effets de l'analyse, se faire payer l'idée même de la satisfaction des désirs en en empêchant la réalisation. Lorsque le surmoi perd de sa force, c'est probablement parce que sa part protectrice s'est révélée insuffisante ou carrément inefficace et cependant, la part critique demeure sévère et intransigeante: l'infléchissement apporté par la tendresse et la bienveillance n'a pas cours. Cette part accusatrice s'alimente sans frein et sans nuance de la tendance (féminine?) à réprimer son agressivité et à la retourner contre le moi. Le soulagement éventuel produit par ce retournement peut à son tour entretenir l'action d'un surmoi tourmenteur dénonçant une intense culpabilité inconsciente voire un besoin de punition. La voie est ouverte alors à toutes les tentatives pour la soulager: le masochisme féminin, bien sûr, et parfois les dérives mélancoliques du masochisme moral.

Si la culpabilité réfère au surmoi, si elle se déclare du fait du retournement de l'agressivité contre le moi, si elle se traduit par la dépendance à «être malade», comment penser que le surmoi au féminin est faible et insuffisant? Cette lacune, lorsqu'elle est source majeure de souffrance psychique, éloigne évidemment de l'ambivalence bien tempérée vis-à-vis de l'objet, certes, mais aussi vis-à-vis du moi, ce serviteur déchiré entre des maîtres en discorde. Le surmoi au féminin pourrait ainsi représenter la forme tyrannique et sévère, la moins aimante, la plus puissante de la conscience morale: peu tolérant, jamais bienveillant, il est embolisé par l'angoisse et ses dérives persécutantes. En ce sens, il peut constituer un destin du féminin parfois dangereux, toujours menaçant.

Ce sont ces stratégies compliquées qui se répètent dans le transfert: de la bisexualité et de ses risques, l'analyse offre l'opportunité de se saisir, pour se défaire de son emprise, pour s'en approprier, même partiellement, et conquérir ainsi «bien plus» de liberté.

\author{
Catherine Chabert \\ catherine.chabert@gmail.com
}

\title{
Note
}

1. Cet article a été présenté lors d une journée clinique organisée par la Société psychanalytique de Québec le 17 octobre 2015..

\section{Références}

Beetschen, A. (2016). Dans Les écueils du féminin. À paraître.

Freud, S. (1896). Projet d'une psychologie, Lettre 52 à Fliess 6-12-1986. Dans Euvres complètes Tome 3 - 1894-1899. Paris: Presses universitaires de France, 2006. 
Freud, S. (1915). Deuil et Mélancolie. Dans Euvres complètes Tome 13 - 1914-1915 (p. 261280). Paris: Presses universitaires de France, 2005.

Freud, S. (1932). 33e Leçon: La féminité. Nouvelle suite des leçons d'introduction à la psychanalyse. Dans Euvres complètes Tome 19 - 1931-1936 (p.195-219). Paris: Presses universitaires de France, 2004.

Freud, S. (1937). L'analyse finie et l'analyse infinie. Dans Euvres complètes Tome 20 - 19371939. Paris: Presses universitaires de France, 2010.

Pontalis, J.-B. (1973). L'insaisissable entre-deux. Nouvelle Revue de psychanalyse (7), 14-23.

Winnicott, D.W. (1966). Clivage des éléments masculins et féminins chez l'homme et chez la femme. Nouvelle Revue de psychanalyse (7), 301-315, 1973. 